\title{
Transient Measurement of Dissolved Oxygen using Membrane Electrodes
}

\author{
Henry Y. Wang \& Xiang-Ming Li* \\ The University of Michigan, Department of Chemical Engineering, Ann Arbor, Michigan \\ 48109-2136, USA
}

(Received 7 April 1988; accepted 6 September 1988)

\begin{abstract}
Transient mode dissolved oxygen measurement has been studied using a specially constructed galvanic membrane electrode controlled by a microcomputer. A four-layer diffusion model was developed to describe the dynamic behavior of the electrode. The data interpretation method is based on on-line computer simulation and parameter estimation techniques. $A$ user-friendly computer software package is also developed for calibration and measurement control. Both experimental and theoretical studies indicate that such measurement is less sensitive to fouling and changes in flow conditions than steady state operation of the electrode.
\end{abstract}

Key words: oxygen sensor, transient mode measurement, diffusion-reaction model, fouling, computer aided measurement.

\section{NOTATION}

A Active surface area of cathode, $\mathrm{cm}^{2}$

$a \quad$ Activity of oxygen

C Oxygen concentration, $\mathrm{gmol} / \mathrm{cm}^{3}$

$D$ Diffusivity of oxygen, $\mathrm{cm} / \mathrm{s}$

F Faraday constant, $9.65 \times 10^{4} \mathrm{col} / \mathrm{gmol}$

$i$ Current output, A

*Present address: South China Institute of Technology, Guangzhou, People's Republic of China

Biosensors 0265-928X/89/\$03.50 (C) 1989 Elsevier Science Publishers Ltd, England. Printed in Great Britain 
L Thickness, cm

$N \quad$ Number of electrons per $\mathrm{O}_{2}$ molecule reduced

$S_{1} \quad$ Solubility of oxygen in $i$ th layer, $g \mathrm{~mol} / \mathrm{cm}^{3} / \mathrm{cm} \mathrm{Hg}$

$t$ time, $s$

$\gamma \quad$ activity coefficient of oxygen

$\chi \quad$ space coordinate, $\mathrm{cm}$

$\begin{array}{ll}\text { Subscripts } \\ i & \text { For } i \text { th layer } \\ s & \text { Bulk solution } \\ \text { ss } & \text { Steady state }\end{array}$

\section{INTRODUCTION}

Dissolved oxygen (DO) is an important measurement parameter in aerobic biological systems. Various DO electrodes with different configurations have been developed (Mancy et al., 1962; Borkowski \& Johnson, 1967; Hemert et al., 1969; Hitchman, 1978; Lee \& Tsao, 1979). One of the problems that needs to be solved for DO measurement in fermentation and waste treatment plants is the measurement error introduced by fouling and varied flow conditions.

Existing electrochemical DO electrodes can be divided into two separate types: galvanic and polarographic. These electrodes consist of an anode and a cathode in contact with an electrolyte. An oxygen permeable membrane is used to cover the electrode. As oxygen diffuses through the membrane and reaches the cathode surface, oxygen reduction may occur if the cathode is at a sufficiently reduced potential with respect to the anode. Depending on the electrode materials used, either an external voltage can be applied as in the case for the polarographic electrodes, or a potential can be generated internally as in the galvanic electrodes.

Two modes of operation, steady state and transient mode, can be used to sample the current output of the electrodes. The steady state mode is based on measuring the steady state current output. This current is directly proportional to the oxygen flux reaching the cathode surface (Aiba et al., 1973). Two main factors can affect the steady state current output. The first is the oxygen concentration in bulk solution which dictates the driving force of the oxygen transport and is the parameter to be measured. The second is the mass transfer resistance which includes the resistance inside the electrode (electrolyte layer, membrane) and outside the electrode (fouling, boundary layer). The electrodes operating in a steady state mode cannot correctly measure the oxygen concentration if the outside resistances, which can 
change, have a significant impact on the oxygen flux. One method to reduce such effects is to use a thicker membrane. However, this will reduce the electrode sensitivity and also increase the response time.

The current transient mode operation is based on pulse voltametric techniques. The polarizing potential is applied as a pulse and the resulting transient current is sampled. Various methods have been used to sample and to interpret the transient current. Mancy (1975) maintained the pulse at a constant time on-time off frequency (time on $=50 \mathrm{~ms}$, time off $=1-3 \mathrm{~s}$ ), and recorded the current when a quasi-steady state condition was reached. Langdon (1984) sampled the current 1.5-3s after the pulse was applied. A recovery time of $3 \mathrm{~min}$ or more was allowed between pulses. The current output showed linear response to the oxygen concentration at certain sampling times (longer than $1.5 \mathrm{~s}$ ). Their experimental results demonstrated that the transient DO measurement was not sensitive to the change in stirring speed.

In our laboratory, a specially constructed galvanic electrode was developed for operation in a transient mode. Instead of using the pulse voltametric technique, a relay controlled by a microcomputer was used to open and close the electrode circuit (Fig. 1). The transient current-time curve was sampled after the circuit was closed. After $1 \mathrm{~s}$, the circuit was opened and the reduction of oxygen at the cathode surface stopped. The oxygen concentration was allowed to be replenished in the electrolyte layer and the membrane for about $1 \mathrm{~min}$. Then another time on-time off cycle began. The oxygen concentrations were determined using the on-line com-

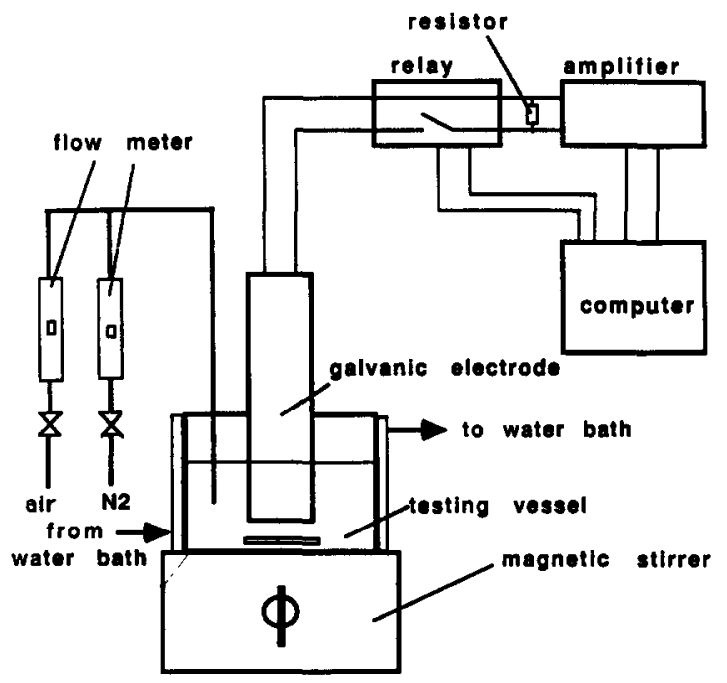

Fig. 1. A schematic diagram of transient DO measurement system using a microcomputer. 
puter simulation and a parameter estimation technique based on the measured current-time curves. Our experimental results showed that such a DO measurement is not sensitive to fouling and changing flow conditions. In theory, the measurement technique is also applicable to other types of membrane electrodes including solid state membrane sensors now under commercial development using microfabrication technology.

\section{TECHNICAL ANALYSIS}

A four-layer model was developed to describe the transient behavior of the electrode. These four layers are: (1) electrolyte, (2) membrane, (3) fouling, and (4) boundary layer. The coordinate system is shown in Fig. 2. One can change the four-layer model into a three-layer model (electrolyte, membrane, boundary layer) in computer simulation by setting the physical properties of the fouling layer and the boundary layer equal to each other. The oxygen transport processes in these four layers are described by the following equations:

$$
\frac{\partial a_{i}}{\partial t}=D_{i} \frac{\partial^{2} a_{i}}{\partial x^{2}} \quad \text { for } i=1,2,3,4
$$

Equation (1) is the Fick's second law applied to these four different layers. The oxygen activity $(a=\gamma C)$ is considered as the transport driving force.

Boundary conditions (BC).

$$
\begin{aligned}
& \frac{a_{i}}{S_{i}}=\frac{a_{i+1}}{S_{i+1}} \quad \text { at } x_{i}, \quad i=1,2,3 \\
& D_{i} \frac{\partial a_{i}}{\partial x}=D_{i+1} \frac{\partial a_{i+1}}{\partial x}, \quad \text { at } x_{i}, \quad i=1,2,3 \\
& a_{4}=\gamma_{4} C_{s}, \quad \text { at } x_{4},
\end{aligned}
$$

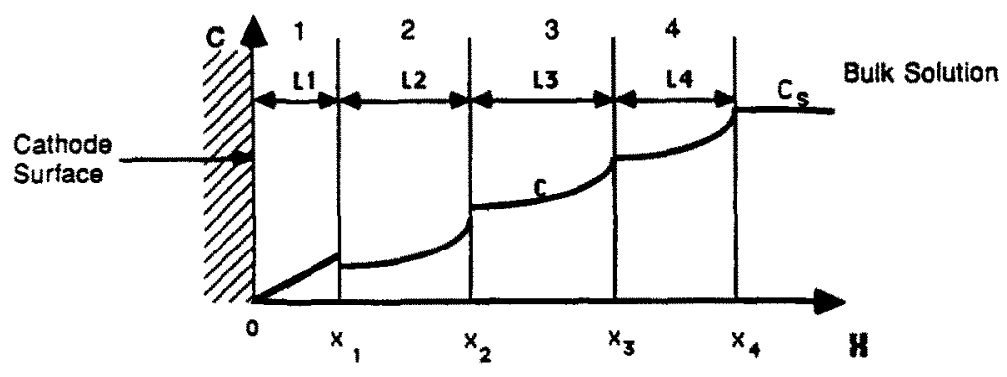

Fig. 2. Four-layer diffusion model for oxygen transport in membrane electrode. (1) Electrolyte layer; (2) teflon membrane; (3) fouling layer; (4) mass transfer boundary layer. 
Equation (2) and eqn (3) are equilibrium and mass conservation conditions at the boundaries between adjacent layers. In eqn (4), the oxygen activity at the outer surface of the boundary layer is assumed equal to the product of the activity coefficient and the bulk concentration

$$
\begin{aligned}
& \text { during time on: } a_{1}=\gamma_{1} C_{1}=0, \quad \text { at } x=0 \\
& \text { during time off: } \frac{\partial a_{1}}{\partial x}=0, \quad \text { at } x=0
\end{aligned}
$$

Equation (5) is the boundary condition at the cathode surface during time on. Since oxygen reduction at the cathode is much faster than the oxygen transport, the oxygen concentration at the cathode surface is assumed to be zero. Equation (6) is the impermeable condition at the cathode surface during time off. In this time period, the oxygen reduction stops, and oxygen concentration in the electrolyte layer and the membrane is replenished

$$
\begin{aligned}
& \text { at } \quad t=0, \\
& a_{4}=\gamma_{4} C_{s} \\
& a_{3}=a_{4} / S_{4} \times S_{3} \\
& a_{2}=a_{3} / S_{3} \times S_{2} \\
& a_{1}=a_{2} / S_{2} \times S_{1}
\end{aligned}
$$

In eqns (7)-(10), the initial oxygen concentrations in these four layers are assumed to be in equilibrium.

Current output equation:

$$
i=F A N D_{1} \frac{\partial a_{1}}{\partial x} \quad \text { at } x=0
$$

At steady state, the following solution can be obtained:

$$
i_{\mathrm{ss}}=\frac{4 F A \gamma_{4} C_{\mathrm{s}}}{\frac{L_{4}}{D_{4}}+\frac{L_{3} S_{4}}{D_{3} S_{3}}+\frac{L_{2} S_{4}}{D_{2} S_{2}}+\frac{L_{1} S_{4}}{D_{1} S_{1}}}
$$

The first and the second terms in the denominator on the right hand side of eqn (12) represent the oxygen transport resistances in the boundary and fouling layers. The third and the fourth terms represent the oxygen transport resistances in the membrane and electrolyte layers. If the first two terms are changed the steady state current output, $i_{\text {ss }}$, will remain essentially unchanged only if

$$
\frac{L_{4}}{D_{4}}+\frac{L_{3} S_{4}}{D_{3} D_{3}} \ll \frac{L_{2} S_{4}}{D_{2} S_{2}}+\frac{L_{1} S_{4}}{D_{1} S_{1}}
$$


This stands for much higher transport resistances in the membrane and electrolyte layers than those in the fouling and boundary layers. In many cases, eqn (13) is hard to satisfy because it will increase the response time significantly. A way to circumvent this difficulty caused by fouling and changing in flow conditions is to operate the electrode in a transient mode. Initially, the oxygen concentrations in the electrolyte layer and membrane reach equilibrium. The concentration profiles are flat as shown schematically in Fig. 3. When the circuit is closed, the oxygen reduction starts to occur, causing a rapid decline in the oxygen concentration profile near the cathode surface. The sampling time only lasts a short period of time before the change in concentration profile reaches the outer edge of the membrane. The circuit is then opened again and followed by a rest period which lasts long enough for oxygen to replenish the electrolyte layer and the membrane. When the electrode is operated in such a transient mode, the oxygen transport resistances outside the membrane will not affect the transient current output. These resistances will only affect the length of the rest period required for replenishing.

\section{HARDWARE}

The cathode was made of a spiral silver wire and coated with epoxy on the back surface to minimize back diffusion. The front surface of the spiral silver cathode was polished to an even curved surface, and then roughened using sandpaper. The membrane was a $1 \mathrm{~mm}$ thick teflon (Dupont FEP, Wilmington, DE), and $1 \mathrm{MK}_{2} \mathrm{HPO}_{4}$ solution was used as the electrolyte. The anode was made of lead. A DASCON-1 interface board (MetraByte,

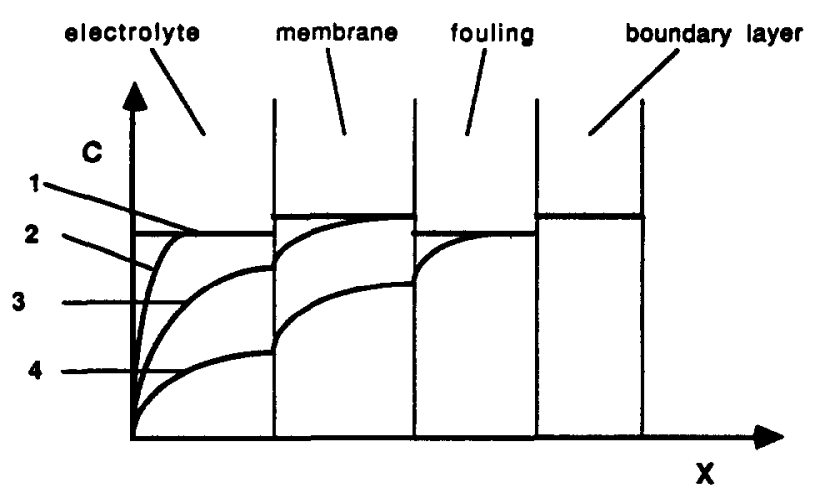

Fig. 3. Changes of oxygen concentration profile during transient measurement. (1) Equilibrium profile; (2) Right after time-on; (3) Concentration decline reaches outer edge of membrane; (4) Concentration decline reaches fouling layer. 
Taunton, MA) with a terminal board (Model STA 01) and a relay board (Model ERA 01) were used for the transient data acquisition. The whole system was controlled by a COMPAQ portable II model 4 microcomputer (COMPAQ Computer Corporation, TX).

\section{SOFTWARE}

We have developed a user friendly software package for this transient DO measurement. The major functions of the software are:

A. Measurements and storages of transient residual current at oxygenfree conditions.

B. Calibrations at different oxygen concentrations and different temperature levels.

C. Transient DO measurements.

The software controls the relay board and the data acquisition board to operate the electrode and sample the current-time curve in a transient mode. All the instructions and operations can be printed on the computer screen according to the function selected.

Function $\mathbf{A}$ is used to measure the residual current of the electrode in an oxygen-free solution. The residual current is sampled in a transient mode at three temperature levels and stored in three files for later use as the base line of the simulated current-time curve.

Function B is used for calibration. During calibration, the electrode is placed in a solution with known oxygen concentration. The current-time curve is sampled in a transient mode. The system parameters, such as the active surface of the cathode, thickness of the electrolyte layer, and the diffusivity of oxygen in the electrolyte will be estimated using a parameter estimation technique (Beck \& Arnold, 1977) with the measured currenttime curve. With an initial set of parameters, computer simulation, which is based on numerically solving eqns (1)-(11) using a Finite Difference Method, will generate a theoretical current-time curve. The simulated current-time curve is obtained by superimposing the residual current to the theoretical current-time curve. The parameter estimation routine is used to search the best parameters which can be used to minimize the least square errors between the simulated results and the measured current-time curve. The best fit parameters will be kept in the data files for further application during measurement. The calibration is performed at three temperature levels. At each temperature level, one to ten concentration settings can be used for calibration.

Function $\mathrm{C}$ is used for DO measurement. During measurement, the 
unknown parameter is the oxygen concentration in the bulk solution.. The system parameter files obtained in calibration will be used for estimating oxygen concentration based on the measured current-time curve. If the temperature in the solution is not the same as the temperature in calibration, the system parameters will be interpolated using second-order Lagrange polynomials according to the temperature.

\section{EXPERIMENTAL RESULTS}

Preliminary experiments were performed in a temperature controlled vessel containing water saturated with a defined gas mixture. Compressed air and nitrogen were mixed in the proper proportion to provide a gas mixture with different oxygen concentrations. The water was bubbled with the gas mixtures and stirred with a magnetic stirrer. The electrode was fixed at a constant location in the vessel. The water level in the vessel remained constant throughout the experiments.

The calibrations were performed at three temperature levels, $30^{\circ} \mathrm{C}, 35^{\circ} \mathrm{C}$, and $40^{\circ} \mathrm{C}$, and six oxygen concentration levels, from $0.01 \mu \mathrm{mol} / \mathrm{ml}$ to $0.23 \mu \mathrm{mol} / \mathrm{ml}$. Eighteen system parameter files were obtained in the form of a data file matrix for interpolation and extrapolation in measurement. Part of the measured current-time curves and the simulated current-time curves are compared and shown in Fig. 4 with good agreement. The DO system was then tested at different oxygen concentrations and different temperature levels. The results are shown in Fig. 5 and Fig. 6.

- The effect of replenishing time (time off) on the transient current output was also studied. Using a replenishing time of $30 \mathrm{~s}$, the gradual drop of the

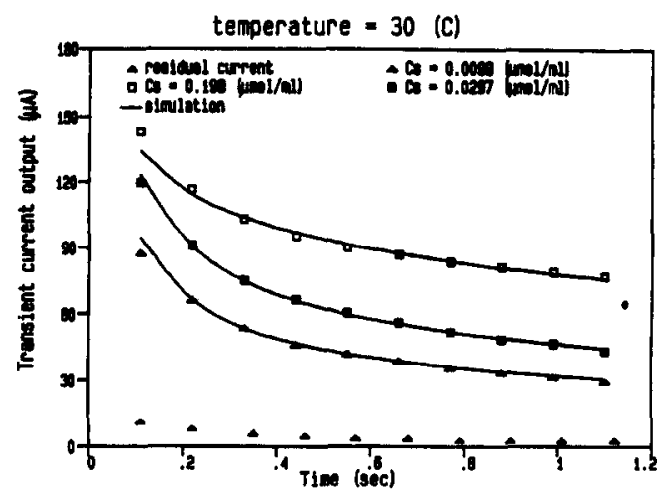

Fig. 4. Experimental transient current output and computer simulation results. 


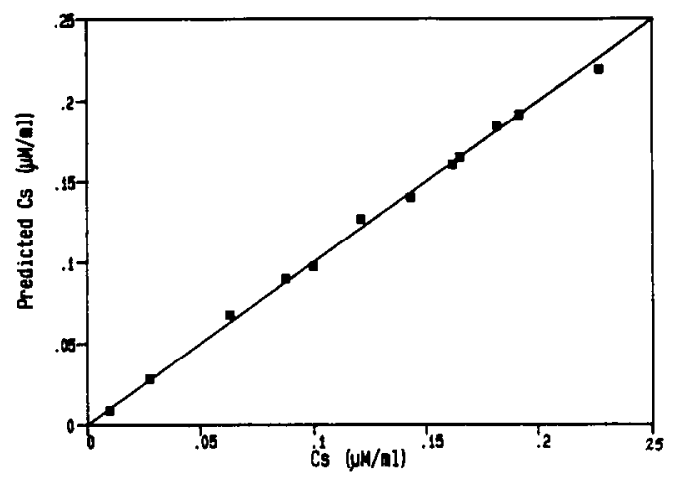

Fig. 5. Transient DO measured values at different oxygen concentrations.

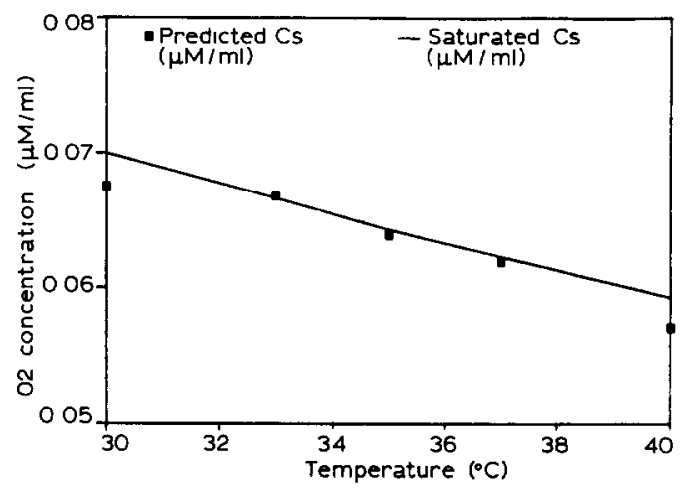

Fig. 6. Transient DO measured values at different temperatures.

current-time curves at sequential sampling cycles indicates that the replenishing time is not long enough to allow the oxygen to reach equilibrium before the next sampling is started (Fig. 7a). Figure $7 \mathrm{~b}$ shows three sequential current outputs with a replenishing time of $60 \mathrm{~s}$. The current-time curves remain unchanged, which indicates the replenishing time is sufficient for accurate measurement in this particular fluid condition. Maintaining a sufficient replenishing time between each sampling is critical for transient DO measurement in extreme fouled conditions. In these conditions fouling will not affect the transient current, but the time required for oxygen reaching equilibrium in the electrolyte layer and the membrane before sampling. Lens paper was used to cover a membrane electrode to simulate fouling. The same electrode was operated under both transient mode and steady state operation to compare the fouling effect. Two replenishing 

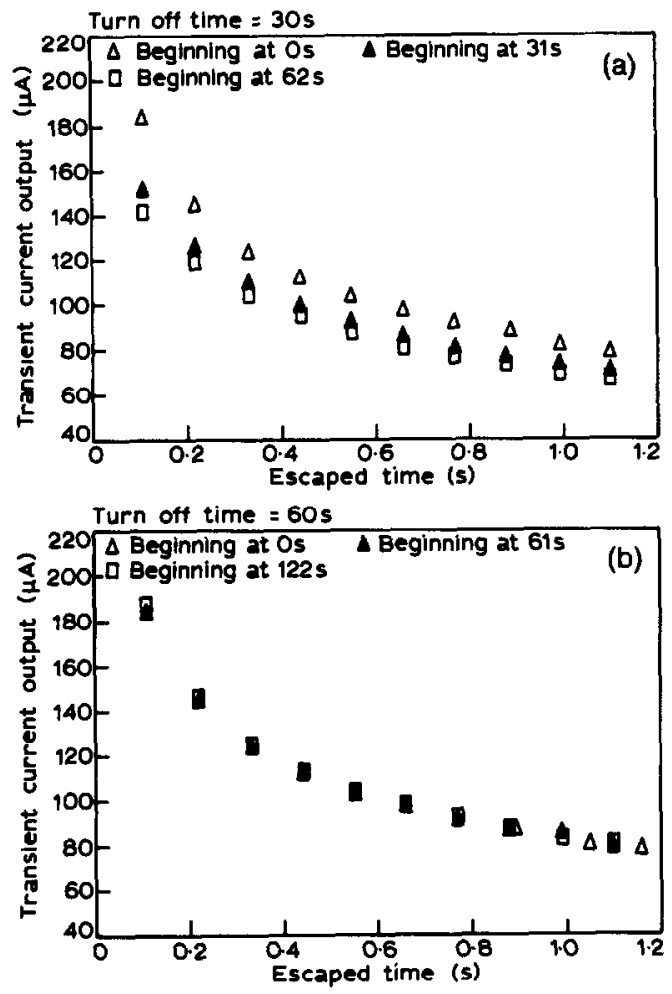

Fig. 7. (a) Time off $=30 \mathrm{~s}$; (b) time off $=60 \mathrm{~s}$.

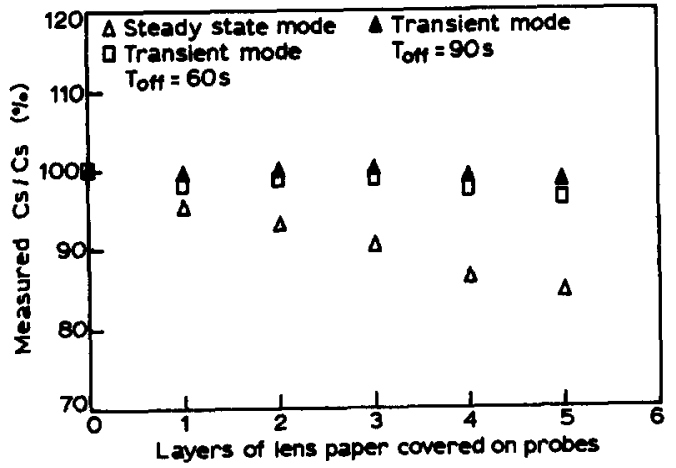

Fig. 8. Effect of simulated fouling by lens papers on transient DO measurement. 


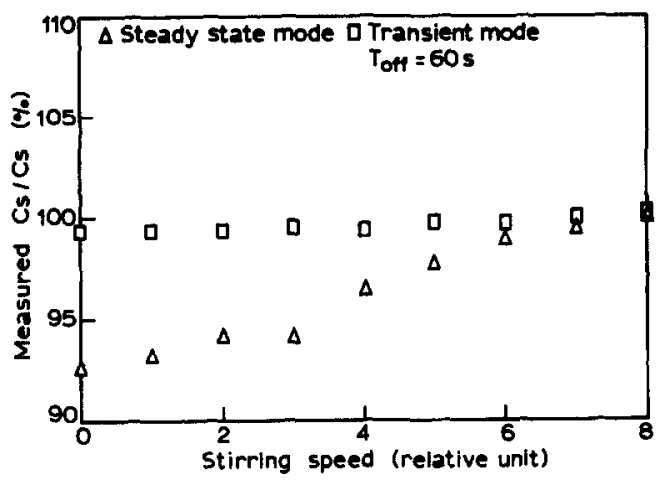

Fig. 9. Effect of stirring speed on transient DO measurement.

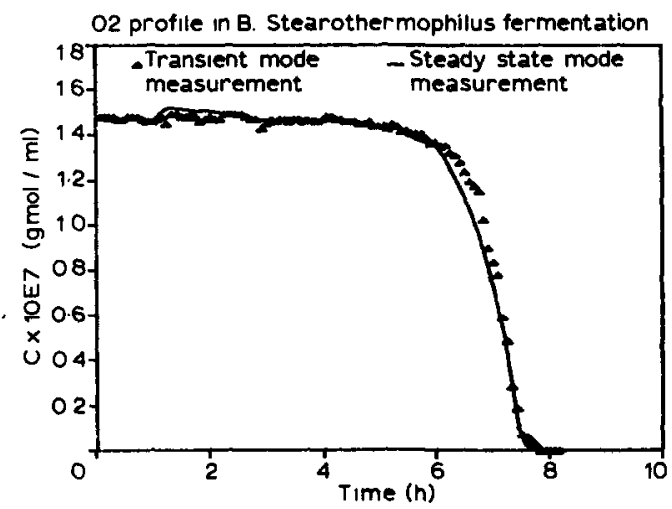

Fig. 10. DO measurement during a Bacillus stearothermophilus fermentation using a starchtryptone medium (temp. $=50^{\circ} \mathrm{C}$ ).

times, 60 s and 90 s, were used in the test. The results are shown in Fig. 8. When five layers of lens paper were covered on the electrode, the oxygen concentration predicted by the steady state measurement was $15 \%$ below the actual concentration. Those predicted by the transient mode measurement were $2 \%$ ( 90 s replenishing time) and $3 \%$ (60s replenishing time). This shows that the transient mode measurement is not sensitive to fouling if the replenishing time is sufficient.

Similarly, effects of varied flow conditions on steady state mode and transient mode measurement were studied using the same electrode to measure oxygen concentration at different stirring speeds. The results are 
shown in Fig. 9. It shows that transient mode measurement is less sensitive to stirring than the steady state mode measurement.

A very viscous fermentation (with $10 \mathrm{wt} \%$ soluble starch) was carried out using Bacillus stearothermophilus in a 2 liter fermentor. The dissolved oxygen was monitored by the regular pseudo-steady state method and the transient mode measurement described above. As shown in Fig. 10, the correlation between the two are extremely good during the fermentation. The drastic change of $\mathrm{DO}$ at $6 \mathrm{~h}$ was adequately measured using both methods. Subsequent uses of the same electrode in this viscous fermentation can only be carried out using the transient mode measurement to obtain accurate DO data. The performance of the oxygen electrode lasts much longer (greater than three months) than a similar electrode under continuous steady state operation.

\section{CONCLUSIONS}

Based on our theoretical analysis and experimental results, we can draw the following conclusions:

(1) A galvanic electrode can also be operated in a transient mode for DO measurement like the transient mode polarographic DO electrodes developed by other researchers.

(2) The membrane electrode operated in a transient mode is less sensitive to fouling caused by the medium and change of flow condition than one operated in a steady state mode. It should be noted that this probe cannot compensate for oxygen consuming organisms fouling the probe, since in such cases the oxygen concentration at the membrane surface can never reach equilibrium.

(3) If the replenishing time is long enough for oxygen to reach equilibrium in the electrolyte layer and the membrane, the fouling (not oxygen consuming) will not affect the transient current output. Fouling will only affect the time required for replenishing.

Our transient DO measurement system requires a microcomputer. It makes the system more complicated than steady state measurement. However, as more and more computers are used in fermentation monitoring and control, the transient DO measurement function can be incorporated into the computer with little modification on both the hardware and software. Alternatively, the logic can be imprinted onto a single chip computer and incorporated inside the electrode circuitry. Currently we are working towards this goal in our research laboratories. 


\section{ACKNOWLEDGEMENTS}

We acknowledge partial financial support from the National Science Foundation (CBT-8406686); X.-M. Li also acknowledges the financial support of a postdoctoral traineeship from the Michigan Biotechnology Institute.

\section{REFERENCES}

Aiba, A. E., Humphrey, A. E. \& Millis, N. F. (1973). Biochemical Engineering, 2nd Edition, Academic Press Inc., New York and London, pp. 327-9.

Beck, J. V. \& Arnold, K. J. (1977). Parameter Estimation in Engineering and Science, John Wiley and Sons, New York.

Borkowski, J. D. \& Johnson, M. J. (1967). Long-lived steam-sterilizable membrane probes for dissolved oxygen measurement. Biotech. Bioeng., 9, 6359.

Hemert, P. Van, Kilburn, D. G., Righelato, R. C. \& Van Wezei, A. L. (1969). A steam-sterilizable electrode of the galvanic type for the measurement of dissolved oxygen. Biotech. Bioeng., 11, 549-60.

Hitchman, M. L. (1978). Measurement of Dissolved Oxygen. John Wiley and Sons, New York.

Langdon, C. (1984). Dissolved oxygen monitoring system using a pulsed electrode: design, performance, and evaluation. Deep-Sea Research, N.S. 31, 11, 1357-67.

Lee, Y. H. \& Tsao, G. T. (1979). Dissolved oxygen electrodes. In Advances in Biochemical Engineering, Vol. 13, ed. T. K. Ghose, A. Flechter and N. Balckebrongh, Springer Verlag, Berlin, pp. 35-86.

Mancy, K. H., (1975). Chemistry and Physics of Aqueous Gas Solutions, ed. W. A. Adams. pp. 281-9.

Mancy, K. H., Okun, D. A. \& Reilley, C. N. (1962). A galvanic cell oxygen analyzer. J. Electroanal. Chem., 4, 65-92. 